PUBLICATION V

\title{
Low-loss converters between optical silicon waveguides of different sizes and types
}

In: IEEE Photonics Technology Letters 2006.

Vol. 18, No. 5, pp. 709-711.

Reprinted with permission from the publisher.

(C) 2006 IEEE 


\title{
Low-Loss Converters Between Optical Silicon Waveguides of Different Sizes and Types
}

\author{
Timo Aalto, Kimmo Solehmainen, Mikko Harjanne, Markku Kapulainen, and Päivi Heimala
}

\begin{abstract}
Two types of low-loss converters between different optical waveguides on silicon-on-insulator are demonstrated. A vertical taper between 9.4- and 3.8- $\mu$ m-thick single-moded rib waveguides gives an excess loss of $0.7 \pm 0.2 \mathrm{~dB}$ with negligible polarization dependency. The second structure converts a 9.7- $\mu \mathrm{m}$-thick rib waveguide into an equally thick and highly multimoded strip waveguide with a negligible loss $(<0.07 \mathrm{~dB})$ for the fundamental mode. The fabrication of both structures is based on a simple two-step etch process with a relaxed mask alignment tolerance and no need for epitaxy.
\end{abstract}

Index Terms-Adiabatic conversion, integrated optics, multistep patterning, silicon microphotonics, silicon waveguide, silicon-oninsulator (SOI) technology, vertical taper.

\section{INTRODUCTION}

$\mathbf{S}$ ILICON-ON-INSULATOR (SOI) is an attractive platform for realizing integrated optical circuits and for integrating them monolithically with control electronics. Due to the ultrahigh refractive index contrast, SOI technology allows the large scale integration of optical circuits. Extreme device miniaturization can be achieved with submicron nanowires [1], while mirrors and groove bends [2] enable the miniaturization of thicker waveguide devices as well. A rib structure provides single-moded (SM) operation even if the waveguide thickness is increased to $>10 \mu \mathrm{m}$ [3]. For low-loss coupling to standard SM fibers the silicon waveguide thickness should be approximately $10 \mu \mathrm{m}$. Some examples of typical cross sections in silicon waveguides are shown in Fig. 1.

The main problem in using thick rib waveguides in optical circuits is the required large bending radius. On the other hand, thin waveguides suffer from inefficient fiber coupling. Thus, the optimum solution would be an adiabatic converter between a thick and a thin waveguide on a single silicon chip. A more general target is an adiabatic conversion between two waveguide cross sections with different sizes and/or types. For example, a rib waveguide could be converted into a strip waveguide with the same or different thickness. This could be used, e.g., to realize compact waveguide arrays, tight bends, waveguide mirrors, or short multimode interference couplers [2].

The above-mentioned conversion structures can be realized by patterning silicon waveguides with more than one etch step. Adiabatic operation is achieved with a sufficiently long conversion structure. Previously, a vertical silicon waveguide taper was demonstrated by using epitaxial growth [4]. However,

Manuscript received January 3, 2006. This work was supported in part by the European Space Agency under ESTEC Contract 17703/03/NL/PA.

The authors are with VTT, Espoo FI-02044, Finland (e-mail: timo.aalto@vtt.fi).

Digital Object Identifier 10.1109/LPT.2006.871150
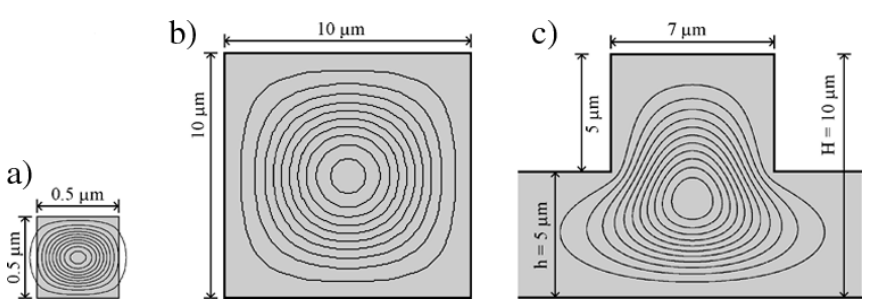

Fig. 1. Example cross sections of different silicon waveguides (not in scale) (a) a nearly SM nanowire, (b) a multimoded strip waveguide, and (c) an SM rib waveguide. Contour lines illustrate the intensity distribution of the fundamental TE mode. Cladding around the (gray) core is not illustrated.

that process was relatively complicated to implement and its details were not disclosed. A rib-strip converter structure has been proposed by others as well [5].

In this study, two multistep waveguide structures were realized in SOI by using two mask layers, two silicon dry etch steps, and no epitaxy. The devices are a low-loss vertical taper and a converter from an SM rib waveguide into an equally thick, highly multimoded strip waveguide. Both devices are simple to fabricate and insensitive to a small misalignment between the masks. The target wavelength is $1550 \mathrm{~nm}$, but the adiabatic devices should operate in a wide spectral range.

\section{Design AND Simulation}

The design of both conversion structures was based on simple linear tapering in the horizontal direction. With two mask layers and two corresponding etch steps, it is possible to create various waveguide cross sections and to connect them with adiabatic converters. Example cross sections along the two chosen conversion structures are shown in Fig. 2 (including the input and output). The calculated mode profiles clearly show the potential for adiabatic conversions between the input and output waveguides - even in the case of finite mask alignment accuracy. Schematic device top views are shown in Fig. 3.

The simulations were carried out by two methods. The local modes corresponding to different waveguide cross sections were solved with a commercial mode solver (TempSelene, v. 4.3). This was used to estimate the impact of both the finite width in the vertical taper's tip and the finite distance of the second etch step at the input end of the multistep structure. Some simulations were also carried out using the three-dimensional beam propagation method (BPM) to provide a rough estimation for the maximum tapering angles for adiabatic operation. The horizontal tapering angles of the vertical taper and the rib-strip converter were chosen to be $0.3^{\circ}$ and $1.7^{\circ}$, respectively. Otherwise the design was primarily based on the modal analysis due to the limited accuracy of the used BPM software (Prometheus v. 


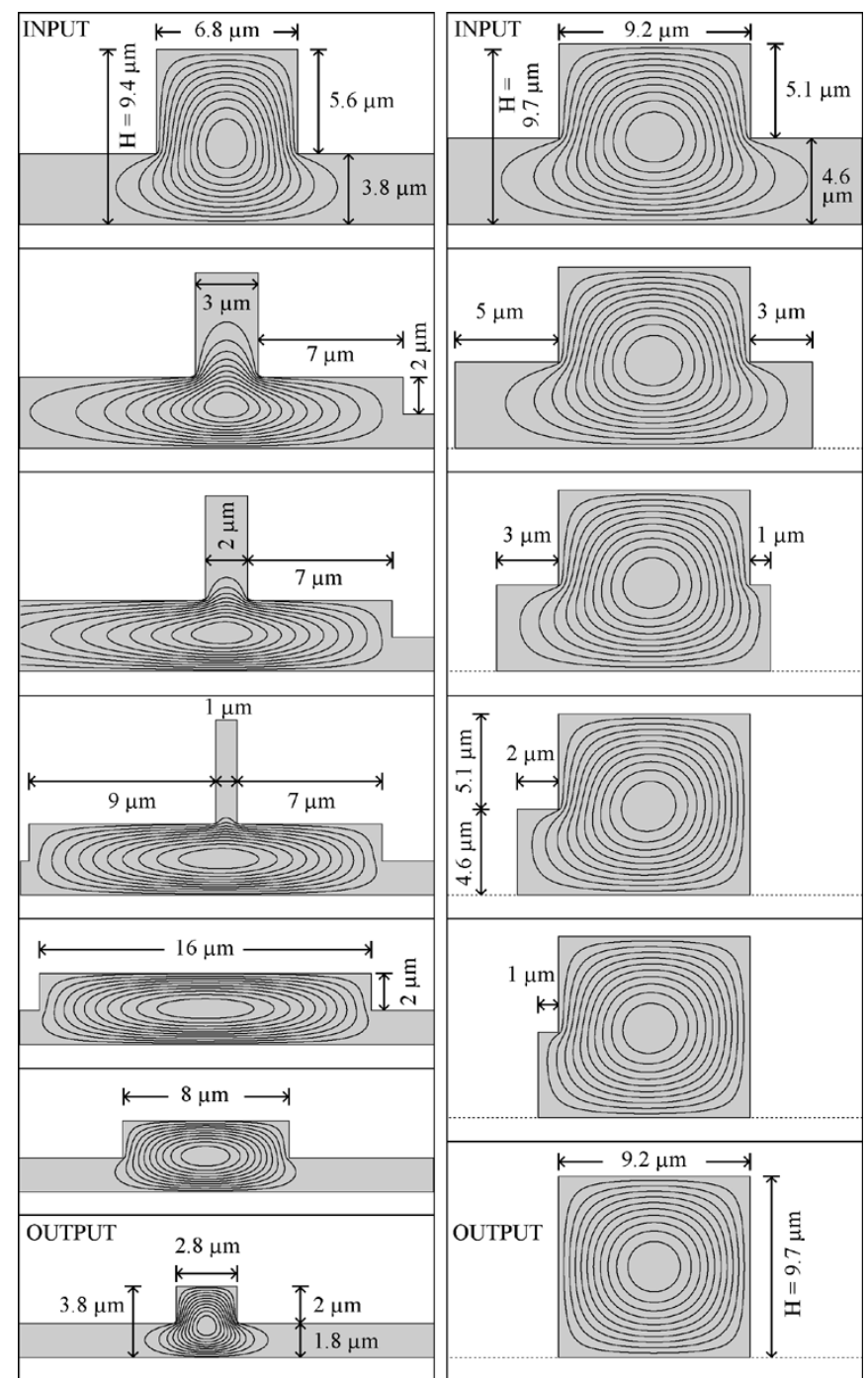

Fig. 2. Different cross sections of the vertical taper (left) and the rib-strip converter (right) with calculated intensity distributions for the fundamental TE mode. The results for TM polarization are almost identical. Misalignment of $1 \mu \mathrm{m}$ is assumed between the two masks. Otherwise dimensions are identical to those measured from the fabricated devices.
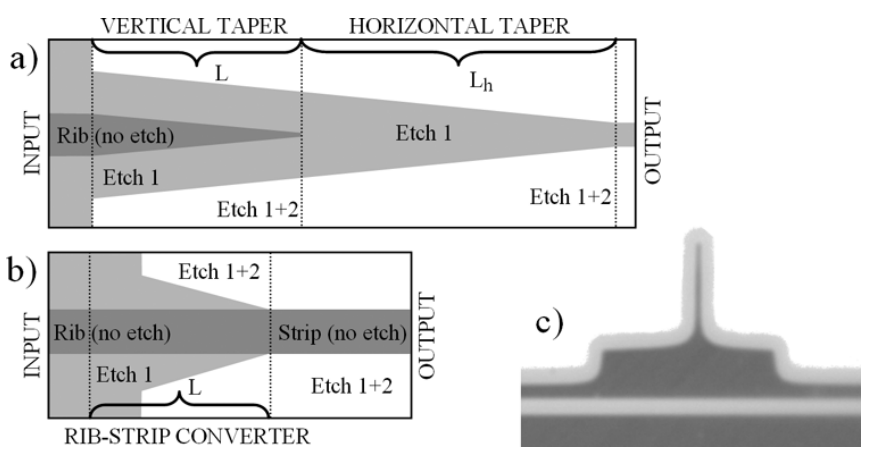

Fig. 3. Schematic top views of (a) the vertical taper and (b) the rib-strip converter. The three colors (dark gray, light gray, and white) illustratate the different silicon thickness after the two etch steps. (c) Microscope image of a fabricated vertical taper's cross section (inverted colors).

4.3). The lengths $(L)$ of the multistep conversion structures were chosen to be 640 and $205 \mu \mathrm{m}$, respectively. The target distance between the two etch steps of the vertical taper was fixed to $8 \mu \mathrm{m}$ and the length $L_{h}$ of the horizontal taper behind the device was set to $1160 \mu \mathrm{m}$. Along the rib-strip converter, the etch step distance is reduced from 4 to $0 \mu \mathrm{m}$. The vertical taper is longer than the rib-strip converter as it induces a larger change into the effective index and the intensity distribution of the fundamental mode.

Special test chips were designed to obtain high measurement accuracy. Each test device converted a thick rib waveguide into a thin rib waveguide or a thick strip waveguide, and back, $N$ times. The output ends of opposite vertical tapers were joined with a 2-mm-long thin rib waveguide. The output ends of opposite rib-strip converters were joined with 0.5 -mm-long strip waveguides. The successive converter pairs were joined with 2-mm-long thick rib waveguides. The input and output waveguides of the vertical taper were designed to be clearly SM, while the input waveguide of the rib-strip converter was designed to operate at the SM-limit. The designs aimed to efficiently suppress the propagation of higher order modes.

Furthermore, each test chip included equally long straight reference waveguides with a cross section identical to the input of the corresponding converter structure.

\section{FABRICATION}

Both devices were fabricated on bonded SOI wafers with 100-mm diameter and $9.5 \pm 0.5 \mu \mathrm{m}$ SOI layer thickness. A $1-\mu \mathrm{m}$-thick oxide hard mask was first deposited on the wafer and then patterned with standard photolithography and mask \#1. The minimum linewidth on the laser-patterned lithographic masks is $900 \mathrm{~nm}$. The primary silicon etch was carried out with an inductively coupled plasma (ICP) etcher by using the patterned oxide as a hard mask. The etch depth was $5.6 \pm 0.3$ and $5.1 \pm 0.3 \mu \mathrm{m}$ for the vertical taper and the rib-strip converter, respectively. This formed the basic rib structure for the input waveguides. Both the original SOI thicknesses and the etch depths involve small variations over the wafer (as indicated above), but these do not disturb the operation of the adiabatic devices.

The secondary silicon etch was also carried out with the ICP etcher, but with a resist mask only. This resist mask was deposited and patterned with the mask \#2 after removing the previous resist layer. The underlying oxide mask patterned with mask \#1 was not removed before the secondary silicon etch. Therefore, the patterned oxide layer below the second resist layer prevented the secondary etching of those areas that were not subject to the primary etch as well. This enabled the passive alignment of the two etch steps in the formation of deep vertical sidewalls for the rib-strip converter. In the lithography equipment used in this work, the misalignment between the two masks can be kept below $1 \mu \mathrm{m}$. The secondary etch depth was $2.0 \pm 0.1 \mu \mathrm{m}$ for the vertical taper and through the remaining $\sim 5 \mu \mathrm{m}$ of SOI for the rib-strip converter.

The remaining resist and oxide layers were then removed and a 1- $\mu$ m-thick thermal oxide was grown on top of the patterned silicon structure to reduce surface roughness and to narrow the finite width of the vertical taper's tip. To minimize the waveguide birefringence, this high-stress oxide was then removed and a low-stress tetraethyl orthosilicate cladding oxide layer of $\sim 1 \mu \mathrm{m}$ was deposited. Finally, the wafers were diced and the 
chip facets were polished to optical quality. A microscope image of a vertical taper's cross section close to the tip is shown in Fig. 3.

\section{Measurement Setup}

In all loss measurements, linearly polarized light at 1550-nm wavelength was coupled from a polarization-maintaining fiber into an input waveguide and then collected from an identical output waveguide into a detector via SM fiber. Index matching oil $(n \approx 1.5)$ was used to reduce the reflections at the waveguide facets. The total insertion loss (IL) of all test devices was calculated by subtracting from the result the power transmitted directly from the input fiber to the output fiber. However, the key to the high measurement accuracy was the comparison of the total IL measured for a test device (with $2 N$ converters) and for its equally long reference waveguide. For the measurement of the on-chip excess loss of a single converter structure this procedure almost completely eliminates the impact of the input and output coupling and, furthermore, reduces the impact of the limited transmission measurement accuracy $( \pm 0.5 \mathrm{~dB})$ by a factor of $2 N$. Due to the slightly increased top surface roughness the propagation loss is higher in the thin rib waveguide than in the reference waveguide, which leads to a worst-case loss estimate for the vertical taper.

The use of SM fibers guarantees that the IL is measured for the fundamental mode only. The polarization-dependent loss (PDL) can be calculated after measuring the excess loss with both TE and TM input polarization.

\section{MEAsuREMENT Results}

The measured total IL for a test device with six successive vertical tapers was 10 and $9.5 \mathrm{~dB}$ for the TE and TM polarization, respectively. For the reference waveguide, the corresponding losses were 5.8 and $5.6 \mathrm{~dB}$, respectively. Thus, the majority of the losses does not originate from the vertical tapering, but from the reflections $(\sim 2 \times 0.8 \mathrm{~dB})$ and the mode mismatch $(\sim 2 \times 1.8 \mathrm{~dB})$ at the nonoptimized waveguide facets, and the propagation loss along the $24-\mathrm{mm}$-long waveguides $(\sim 0.5 \mathrm{~dB})$. Based on the results, the estimated excess loss for a single vertical taper is $0.7 \pm 0.1 \mathrm{~dB}$ for TE and $0.65 \pm 0.1 \mathrm{~dB}$ for TM. Therefore, the PDL is below the measurement accuracy and the excess loss can be estimated as $0.7 \pm 0.2 \mathrm{~dB}$ including the impact of the PDL.

Similarly, the measured total IL for 22 successive rib-strip converters was 5.4 and $5.2 \mathrm{~dB}$ for TE and TM polarization, respectively. For the reference waveguide, the losses were 4.4 and
$5.3 \mathrm{~dB}$, respectively. The excess loss for a single rib-strip converter is $0.045 \pm 0.02 \mathrm{~dB}$ for TE and $-0.005 \pm 0.02 \mathrm{~dB}$ for TM. The apparently negative loss for TM is due to the finite measurement accuracy. In conclusion, the excess loss and the PDL are both below $0.07 \mathrm{~dB}$ for a single rib-strip converter.

\section{CONCLUSION}

Two types of waveguide conversion structures were successfully demonstrated on SOI. An SM vertical taper provided waveguide thickness conversion from 9.4 to $3.8 \mu \mathrm{m}$ with an excess loss of $0.7 \pm 0.2 \mathrm{~dB}$. The other device converted a 9.7- $\mu \mathrm{m}$-thick rib waveguide into an equally thick and highly multimoded strip waveguide with a negligible loss $(<0.07 \mathrm{~dB})$. Both devices were fabricated with a simple process involving two masks and two Si etch steps.

In the future, the device geometries should be further optimized with respect to both the excess loss and the device size. Based on the simulations, it is expected that, e.g., nonlinear tapering can reduce the tapering loss. Other waveguide thicknesses and other types of conversions should also be tested.

The applicability of the vertical taper is mostly limited by the thickness variation of the down-tapered waveguide. However, vertical tapers can also be used on a separate input coupling chip with an array of SM fiber pigtails on one side. A dense array of thin Si waveguides on the opposite side can then be used to couple light into various optical devices that would otherwise have high fiber-coupling losses. With antireflection coatings and optimized cross sections at the ends of the input and output waveguides the reflections and mode mismatch losses can be minimized and the main remaining loss element is the vertical taper itself.

\section{REFERENCES}

[1] Y. A. Vlasov and S. J. McNab, "Losses in single-mode silicon-on-insulator strip waveguides and bends," Opt. Express, vol. 12, pp. 1622-1631, 2004.

[2] T. Aalto, P. Heimala, S. Yliniemi, M. Kapulainen, and M. Leppihalme, "Fabrication and characterization of waveguide structures on SOI," in Proc. SPIE, vol. 4944, 2003, pp. 183-194.

[3] R. A. Soref, J. Schmidtchen, and K. Petermann, "Large single mode rib waveguides in GeSi-Si and Si-on-SiO2," IEEE J. Quantum Electron., vol. 27, no. 8, pp. 1971-1974, Aug. 1991.

[4] I. Day, I. Evans, A. Knights, F. Hopper, S. Roberts, J. Johnston, S. Day, J. Luff, H. Tsang, and M. Asghari, "Tapered silicon waveguides for low insertion loss highly-efficient high-speed electronic variable optical attenuators," in Proc. OFC 2003, Atlanta, GA, 2003, pp. 249-251.

[5] D. Dai, J. He, and S. He, "Elimination of multimode effects in a silicon-on-insulator etched diffraction grating demultiplexer with lateral tapered air slots," IEEE J. Sel. Topics Quantum Electron., vol. 11, no. 2, pp. 439-443, Mar./Apr. 2005. 